\title{
Patterns of fruit and vegetable consumption among Iranian adults: a SuRFNCD-2007 study
}

\author{
Alireza Esteghamati*, Sina Noshad, Arash Nazeri, Omid Khalilzadeh, Mohammad Khalili and \\ Manouchehr Nakhjavani \\ School of Medicine, Endocrinology and Metabolism Research Center (EMRC), Vali-Asr Hospital, Tehran University of Medical \\ Sciences, PO Box 13145-784, Tehran, Iran
}

(Submitted 19 January 2011 - Final revision received 8 August 2011 - Accepted 4 September 2011 - First published online 10 October 2011)

\begin{abstract}
The aim of the present study was to describe the patterns of fruit and vegetable (F\&V) intake in a nationally representative sample of the Iranian population. The data collected in the Third National Surveillance of Risk Factors of Non-communicable Diseases (SuRFNCD-2007) were used. In a sample of 3702 Iranian adult participants, patterns of F\&V consumption were assessed using the WHO STEPwise method. Low F\&V consumption was defined as intake of less than five servings of fruit and/or vegetable daily according to the WHO guidelines. F\&V consumption was compared among different age groups, sex and urban/rural areas using complex sample analysis. On average, $1 \cdot 26$ servings of fruit and 1.32 servings of vegetables were consumed daily. Taken together, Iranian adults consumed $2.58 \mathrm{~F} \& \mathrm{~V}$ servings per $\mathrm{d}$, with females eating more than males $(P \leq 0 \cdot 001)$. Moreover, there was a trend towards lower consumption rates in older-age categories $(P=0.003)$. Prevalence of low F\&V intake (less than five servings daily) was $87.5 \%$ and also tended to be higher in older-age categories $(P=0.004)$. Prevalence of low intake did not differ significantly among men and women or urban and rural areas. A high prevalence of low F\&V consumption in the Iranian adult population was documented. These findings may guide health policy makers in developing specific plans to encourage adequate $\mathrm{F} \& \mathrm{~V}$ intake.
\end{abstract}

Key words: Fruit: Vegetables: BMI

Fruit and vegetable (F\&V) consumption has long been shown to be associated with a decrease in all-cause mortality, incidence of CVD, stroke and certain types of cancer ${ }^{(1-4)}$. Although not universally accepted, increased F\&V intake has also been suggested to be associated with a lower incidence of diabetes and obesity ${ }^{(5,6)}$.

According to the WHO panel on diet, nutrition and prevention recommendations, an intake of five or more servings of $\mathrm{F} \& \mathrm{~V}$ per $\mathrm{d}$ ( $400 \mathrm{~g}$ or more with $80 \mathrm{~g}$ servings) is considered adequate consumption $^{(7)}$. This value has served as a criterion for adequate $F \& V$ intake in a number of studies ${ }^{(8)}$. Despite the great impact of $\mathrm{F} \& \mathrm{~V}$ consumption on public health, the pattern of $F \& V$ intake has not been evaluated by a nation-wide survey in the Iranian adult population. In the present study, we aimed to investigate the pattern of F\&V consumption in the Iranian adult population.

\section{Subjects and methods}

Study population

The present study uses the data collected in the Third National Surveillance of Risk Factors of Non-communicable
Diseases in Iran (SuRFNCD-2007). Details of the survey can be found elsewhere ${ }^{(9)}$. In brief, a cluster sampling scheme was applied to randomly select a representative sample of the Iranian adult population across the country. Trained health care professionals conducted household interviews and physical examinations. All interviews were in Persian. The data were recorded in standardised questionnaires and were rechecked based on a predetermined schedule. The study was conducted according to the guidelines laid down in the Declaration of Helsinki and the study protocol was approved by the Iran Center for Disease Control Ethics Committee. Informed consent was obtained from all subjects before the interview and was witnessed and formally recorded by the interviewer.

\section{Fruit and vegetable data}

Participants were asked to answer the following four questions regarding their consumption of $F \& V$ :

(1) In a typical week, on how many days do you eat fruit?

Abbreviations: F\&V, fruit and vegetables; SuRFNCD, Surveillance of Risk Factors of Non-communicable Diseases.

*Corresponding author: Professor A. Esteghamati, fax +98 21 64432466, email esteghamati@tums.ac.ir 
(2) How many servings of fruit do you eat on one of those days?

(3) In a typical week, on how many days do you eat vegetables?

(4) How many servings of vegetables do you eat on one of those days?

A serving size was defined as $80 \mathrm{~g}$ of fruit or vegetables. In detail, one serving of vegetables was defined as one cup of raw green leafy vegetables, one-half cup if cooked or chopped or one-half cup of vegetable juice. Similarly, one medium-sized fruit (such as banana, apple, orange, etc.), one-half cup if cooked, chopped or canned, or one-half cup of fruit juice (any type) was defined as one serving of fruit. Tubers including potato, sweet potato and beet were not included. To guide the respondents through answering, cups with different serving sizes and show cards (containing pictures of various servings of $\mathrm{F} \& \mathrm{~V}$ ) were prepared beforehand and were shown while answering questions. Average daily serving size of fruit/vegetables was calculated according to the STEPS guidelines as follows ${ }^{(10)}$ : number of days fruit (or vegetables) being eaten in a typical week multiplied by number of servings eaten in a day divided by 7 .

\section{Statistical analysis}

Complex sample survey analysis was performed using SPSS 17.0 for Windows (2008; Chicago, IL, USA). For extrapolation of the results to the Iranian adult population, data were weighted for age (10-year strata), sex and residence area (rural/urban) using Iran's 2006 national census estimates (according to the census estimates, there were 31409737 adults aged 25-64 years residing in $\operatorname{Iran}^{(11)}$ ). Based on the age, sex and residential area strata, clusters of the sampling protocol, and the determined weights, an analysis plan was established. Continuous variables were compared across sex, residential area and age groups using general linear models, and categorical variables were compared using designbased $\chi^{2}$ analysis. $P$ values for linear trend were calculated when comparing across age groups. National estimates are expressed as means with their standard errors for continuous variables. In all analyses, a $P$ value $<0.05$ was considered statistically significant.

\section{Results}

A total of 4231 adults aged 25-64 years were enrolled in the present study. However, some data for fruit ( $n$ 193), vegetable ( $n$ 215) and F\&V ( $n$ 121) consumption were not available. After excluding participants with missing data ( $n$ 529; complete response rate $87.5 \%$ ), patterns of $\mathrm{F} \& \mathrm{~V}$ consumption were investigated in 3702 subjects. Characteristics of the survey's participants have been described elsewhere ${ }^{(12)}$. Average age of the study participants was 43.5 years, of which $50 \%$ were women and $68.4 \%$ resided in urban areas. Table 1 summarises the patterns of $F \& V$ consumption. Iranian adults con-

Table 1. Fruit and vegetable consumption among the Iranian adult population

(Mean values with their standard errors)

\begin{tabular}{|c|c|c|c|c|c|c|}
\hline & \multicolumn{2}{|c|}{$\begin{array}{l}\text { Number of days } \\
\text { consumption in a } \\
\text { typical week }\end{array}$} & \multirow[b]{2}{*}{$P$ trend } & \multicolumn{2}{|c|}{$\begin{array}{l}\text { Consumed servings } \\
\text { on average } \\
\text { per d }\end{array}$} & \multirow[b]{2}{*}{$P$ trend } \\
\hline & Mean & SEM & & Mean & SEM & \\
\hline \multicolumn{7}{|l|}{ Fruit consumption } \\
\hline \multicolumn{7}{|l|}{ Age (years) } \\
\hline $25-34(n 979)$ & 4.32 & $0 \cdot 10$ & 0.024 & 1.31 & 0.05 & $<0.001$ \\
\hline $35-44(n 975)$ & 4.41 & $0 \cdot 10$ & & $1 \cdot 27$ & 0.05 & \\
\hline $45-54(n 930)$ & $4 \cdot 13$ & 0.10 & & 1.16 & 0.05 & \\
\hline $55-64(n 818)$ & 4.02 & 0.11 & & $1 \cdot 10$ & 0.04 & \\
\hline \multicolumn{7}{|l|}{ Sex } \\
\hline Males $(n 1851)$ & $4 \cdot 18$ & 0.07 & 0.091 & 1.22 & 0.03 & 0.415 \\
\hline Females $(n 1851)$ & 4.38 & 0.08 & & 1.28 & 0.05 & \\
\hline \multicolumn{7}{|l|}{ Residential area } \\
\hline Urban (n 2532) & 4.52 & 0.06 & $<0.001$ & 1.33 & 0.03 & $<0.001$ \\
\hline Rural ( $n 1170)$ & 3.65 & 0.08 & & 1.05 & 0.05 & \\
\hline Total national estimate ( $n$ 3702) & 4.33 & 0.06 & & 1.26 & 0.03 & \\
\hline \multicolumn{7}{|l|}{ Vegetable consumption } \\
\hline \multicolumn{7}{|l|}{ Age (years) } \\
\hline $25-34$ (n 979) & 4.56 & 0.08 & 0.008 & 1.28 & 0.04 & 0.070 \\
\hline $35-44(n 975)$ & 4.76 & $0 \cdot 10$ & & 1.37 & 0.06 & \\
\hline $45-54(n 930)$ & 4.87 & 0.09 & & 1.37 & 0.05 & \\
\hline $55-64(n 818)$ & 4.58 & $0 \cdot 10$ & & 1.23 & 0.04 & \\
\hline \multicolumn{7}{|l|}{ Sex } \\
\hline Males ( $n$ 1851) & 4.49 & 0.07 & $<0.001$ & 1.24 & 0.04 & 0.002 \\
\hline Females ( $n$ 1851) & 4.87 & 0.07 & & 1.40 & 0.04 & \\
\hline \multicolumn{7}{|l|}{ Residential area } \\
\hline Urban (n 2532) & 4.68 & 0.05 & 0.917 & 1.29 & 0.03 & 0.075 \\
\hline Rural $(n 1170)$ & 4.69 & 0.12 & & 1.40 & 0.05 & \\
\hline Total national estimate ( $n$ 3702) & 4.68 & 0.05 & & 1.32 & 0.03 & \\
\hline
\end{tabular}


Table 2. Consumed servings of fruit and vegetables (F\&V) on average per $d$ among the Iranian adult population

(Percentage of prevalence, $95 \%$ confidence intervals, mean values with their standard errors)

\begin{tabular}{|c|c|c|c|c|c|c|c|c|c|c|}
\hline & & & & & \multicolumn{6}{|c|}{ Daily servings } \\
\hline & \multicolumn{4}{|c|}{ Daily F\&V intake } & \multicolumn{3}{|c|}{$<5$} & \multicolumn{3}{|c|}{$\geq 5$} \\
\hline & Mean & SEM & $P$ trend & $\begin{array}{l}\text { Estimated } \\
\text { population } \\
\text { (millions) }^{\star}\end{array}$ & Prevalence \% & $95 \% \mathrm{Cl}$ & $\begin{array}{c}\text { Estimated } \\
\text { population } \\
\text { (millions) }\end{array}$ & Prevalence \% & $95 \% \mathrm{Cl}$ & $P$ trend \\
\hline \multicolumn{11}{|l|}{ Age (years) } \\
\hline $25-34(n 979)$ & $2 \cdot 60$ & 0.08 & 0.003 & 11 & $86 \cdot 3$ & $83 \cdot 0,89 \cdot 1$ & $1 \cdot 8$ & $13 \cdot 7$ & $10 \cdot 9,17 \cdot 0$ & 0.004 \\
\hline $35-44(n 975)$ & 2.65 & 0.10 & & $7 \cdot 8$ & $87 \cdot 0$ & $83.5,89.8$ & $1 \cdot 2$ & $13 \cdot 0$ & $10 \cdot 2,16 \cdot 5$ & \\
\hline $45-54(n 930)$ & 2.52 & 0.07 & & 5.5 & 88.7 & $85 \cdot 8,91 \cdot 1$ & 0.7 & $11 \cdot 3$ & $8 \cdot 9,14 \cdot 2$ & \\
\hline $55-64(n 818)$ & $2 \cdot 34$ & 0.06 & & $3 \cdot 1$ & $91 \cdot 2$ & $89 \cdot 4,92 \cdot 7$ & 0.2 & $8 \cdot 8$ & $7 \cdot 3,10 \cdot 6$ & \\
\hline \multicolumn{11}{|l|}{ Sex } \\
\hline Males ( $n$ 1851) & 2.47 & 0.06 & 0.040 & $14 \cdot 1$ & 88.4 & $86 \cdot 0,90 \cdot 5$ & $1 \cdot 8$ & $11 \cdot 6$ & $9 \cdot 5,14 \cdot 0$ & 0.274 \\
\hline Females $(n 1851)$ & $2 \cdot 68$ & 0.07 & & $13 \cdot 4$ & $86 \cdot 6$ & $84 \cdot 0,88 \cdot 8$ & $2 \cdot 1$ & 13.4 & $11 \cdot 2,16 \cdot 0$ & \\
\hline \multicolumn{11}{|l|}{ Residential area } \\
\hline Urban (n 2532) & $2 \cdot 63$ & 0.05 & 0.110 & $19 \cdot 7$ & $87 \cdot 3$ & $85 \cdot 3,89 \cdot 1$ & $2 \cdot 8$ & $12 \cdot 7$ & $10 \cdot 0,14 \cdot 7$ & 0.741 \\
\hline Rural ( $n 1170)$ & 2.46 & 0.09 & & $7 \cdot 8$ & 87.9 & $84.5,90.7$ & $1 \cdot 1$ & $12 \cdot 1$ & $9 \cdot 3,15 \cdot 5$ & \\
\hline $\begin{array}{l}\text { Total national estimate } \\
\quad(n \text { 3702) }\end{array}$ & 2.58 & 0.05 & & $27 \cdot 5$ & $87 \cdot 5$ & $85 \cdot 8,89 \cdot 1$ & $3 \cdot 9$ & $12 \cdot 5$ & $10 \cdot 9,14 \cdot 2$ & \\
\hline
\end{tabular}

${ }^{\star}$ Rounded to the nearest million.

sumed fruit, on average, in $4.33 \mathrm{~d} /$ week, with younger age categories and urban dwellers consuming more often $(P=0.024$ and $<0.001$, respectively). No sex predilection was observed in this regard $(P=0 \cdot 091)$. Iranian adults reported consuming on average $1 \cdot 26$ daily servings of fruit, with younger and urban dwellers consuming more $(P<0.001$ for both). The number of fruit servings did not differ significantly between the two sexes $(P=0.415)$. About $31.6 \%$ of the subjects responded that they eat fruit every day, for vegetable intake this estimate was $38.1 \%$.

Iranian adults had vegetables on their daily diet on average $4 \cdot 68 \mathrm{~d} /$ week. Women tended to eat vegetables more frequently compared with men $(4 \cdot 49 \mathrm{~d} /$ week for men and $4.87 \mathrm{~d} /$ week for women, $P<0.001)$. Although subjects in the 55-64 years age category consumed vegetables less frequently than the $45-54$ years age group, there was an overall incremental trend in vegetable consumption across age categories $(P$ for trend $=0 \cdot 008)$. Frequency of vegetable consumption was not significantly different in urban $v$. rural areas $(P=0.917)$. The mean number of servings of vegetables eaten per $\mathrm{d}$ was 1.32 and did not differ significantly among age and urban/rural categories $(P=0.070$ and 0.075 , respectively). On average, women consumed more servings of vegetables per $\mathrm{d}$ compared with men ( 1.40 for women and 1.24 for men, $P=0.002$ ).

Taken together, Iranian adults ate on average 2.58 servings of F\&V daily. F\&V consumption significantly decreased with increasing age ( $P=0.004)$, albeit the $35-44$ years age category did not follow this general trend. Women consumed more amounts of $\mathrm{F} \& \mathrm{~V}(P=0.040)$; no significant difference was observed between urban and rural residents (Table 2).

Based on the WHO definition on low F\&V consumption, about $87.5 \%$ (95\% CI $85.8,89 \cdot 1)$ of the subjects consumed less than five servings of $\mathrm{F} \& \mathrm{~V}$ per $\mathrm{d}$. Low fruit consumption tended to be higher in older age categories $(P=0 \cdot 004)$.
The prevalence of low fruit consumption was not significantly different among men and women or rural and urban dwellers ( $P=0.274$ and $P=0.741$, respectively)

\section{Discussion}

The present study demonstrated that average daily F\&V consumption was 2.58 servings (206g) per $\mathrm{d}$ among the Iranian adult population. Recently, two regional observations in Iran have reported daily F\&V intakes of 1.76 (mean age 64 years) and 5.6 (mean age $37 \cdot 2$ years) ${ }^{(13,14)}$. Since these studies are not conducted on a national scale, and the age categories of the studied populations are different from the present study, a direct comparison with the present results is not possible.

About $87.5 \%$ of Iranian adults consumed less than the WHO-recommended daily intake (88.4\% of men and $86.6 \%$ of women). In the Middle East region, Iran stands somewhere in the middle; with a lower consumption than Turkey ( 80.7 for men and 81.3 for women) and the United Arab Emirates (77.8 for men and 74.5 for women), and higher than its eastern neighbour Pakistan (99.2 for men and $99 \cdot 3$ for women) ${ }^{(15)}$ Comparing with developed nations, Iran has a lower F\&V consumption. For instance, reports from the USA indicate a low consumption rate of $77 \%(1991)$ and $75.3 \%(2003)^{(16,17)}$. Observed estimates for the UK ( $78 \%$ for men and $74 \%$ for women) and Spain ( $78 \%$ for men and $75.1 \%$ for women) are also in the same range ${ }^{(15,18)}$. On the other hand, only $46 \%$ of men and $47 \%$ of women in France consume less than five units of $F \& V$ daily ${ }^{(19)}$. Due to different sampling methods applied in each of these studies, direct comparisons should be done with caution.

In the present study, we showed that there is a linear trend towards low F\&V consumption in advanced age groups, an observation made by other research groups in both developed 
and developing countries ${ }^{(15,20)}$. A multi-site study (INDEPTH), conducted in largely rural areas of south and south-eastern Asia, using the WHO STEPwise approach, showed that the increase in age is linked to low F\&V consumption, with the oldest group having the lowest intake ${ }^{(21)}$. Some studies, however, have pointed to a reverse correlation between age and low F\&V consumption ${ }^{(22,23)}$.

Similar to the present results, previous studies have also reported inconsistent results regarding sex differencecs in F\&V consumption. For example, a nationally representative study in the USA reported that in four out of the six centres studied, there were little differences with respect to $\operatorname{sex}^{(24)}$. The results of the INDEPTH study indicated that the rate of low F\&V consumption is higher in women ${ }^{(21)}$, while some other reports have observed that the rates are higher in men ${ }^{(24,25)}$. In the World Health Survey, among the fifty-two studied countries, only in fifteen countries were there sex differences in the pattern of $F \& V$ intake, of which in five countries, women were consuming less and in ten, consuming more $^{(15)}$. We should also note that according to the World Health Survey, in forty-one countries, F\&V consumption did not differ between urban and rural areas; similar observations were replicated in the present study ${ }^{(15)}$.

Potential limitations in the present study should be acknowledged. We used a questionnaire-based method for quantification of $\mathrm{F} \& \mathrm{~V}$ consumption, relying solely on the respondents' memory. This could cause measurement error and skew the results towards over- or underestimation. Nevertheless, it should be noted that the questionnaire used in this survey was approved by the WHO and covered questions only about the past few days before the interview. Moreover, interviewers provided respondents with images and examples of $\mathrm{F} \& \mathrm{~V}$ servings, adding to the accuracy of quantifying $\mathrm{F} \& \mathrm{~V}$ intake.

In summary, the present study gives insight about the magnitude of low F\&V consumption problem. Iranian's F\&V intake is remarkably low, lower than some of the developing and most of the developed nations. Of every eight adults living in Iran, seven have inadequate $F \& V$ intake with respect to the WHO guidelines, and this is a pivotal - yet preventable - source of morbidity and mortality. Plans have to emerge to promote adequate F\&V consumption; policy makers, public health personnel, mass media and the educational system are key players in this regard.

\section{Acknowledgements}

This study received no specific grant from any funding agency in the public, commercial or non-profit sectors. A. E. provided clinical expertise throughout the project. S. N. and A. N. performed the statistical analysis and drafted the manuscript. O. K. supervised the analysis and manuscript preparation. M. K. contributed to the literature review and helped with the revised manuscript. M. N. provided clinical expertise and also helped with the revised manuscript. All authors read and approved the final manuscript draft. The authors declare that they have no conflict of interest.

\section{References}

1. Key TJ (2011) Fruit and vegetables and cancer risk. Br J Cancer 104, 6-11.

2. Bazzano LA, He J, Ogden LG, et al. (2002) Fruit and vegetable intake and risk of cardiovascular disease in US adults: the first National Health and Nutrition Examination Survey Epidemiologic Follow-up Study. Am J Clin Nutr 76, 93-99.

3. He FJ, Nowson CA \& MacGregor GA (2006) Fruit and vegetable consumption and stroke: meta-analysis of cohort studies. Lancet 367, 320-326.

4. Agudo A, Cabrera L, Amiano P, et al. (2007) Fruit and vegetable intakes, dietary antioxidant nutrients, and total mortality in Spanish adults: findings from the Spanish cohort of the European Prospective Investigation into Cancer and Nutrition (EPIC-Spain). Am J Clin Nutr 85, $1634-1642$

5. Bazzano LA, Li TY, Joshipura KJ, et al. (2008) Intake of fruit, vegetables, and fruit juices and risk of diabetes in women. Diabetes Care 31, 1311-1317.

6. Alinia S, Hels O \& Tetens I (2009) The potential association between fruit intake and body weight - a review. Obes Rev 10, 639-647.

7. World Health Organization/FAO (2003) Diet, Nutrition and the Prevention of Chronic Diseases; Report of a Joint WHO/ FAO Expert Consultation. Geneva: WHO.

8. Liu S, Manson JE, Lee IM, et al. (2000) Fruit and vegetable intake and risk of cardiovascular disease: the Women's Health Study. Am J Clin Nutr 72, 922-928.

9. CDC of Iran (2008) The Profile of the Third National Surveillance of Noncommunicable Disease Risk Factors in the IR of Iran. Tehran: Center for Disease Control, Ministry of Health \& Medical Education.

10. World Health Organization (2005) WHO STEPS: Chronic Disease Risk Factor Surveillance: Analysis Programs Documentation. Geneva: WHO.

11. Statistical Center of Iran (2006) Iran Population Estimates 2006. http://www.sci.org.ir/portal/faces/public/sci/sci.gozide/sci. PopEstimate (accessed April 2010)

12. Esteghamati A, Meysamie A, Khalilzadeh O, et al. (2009) Third national Surveillance of Risk Factors of Non-Communicable Diseases (SuRFNCD-2007) in Iran: methods and results on prevalence of diabetes, hypertension, obesity, central obesity, and dyslipidemia. BMC Public Health 9, 167.

13. Mirmiran P, Noori N, Zavareh MB, et al. (2009) Fruit and vegetable consumption and risk factors for cardiovascular disease. Metabolism 58, 460-468.

14. Salehi L, Eftekhar H, Mohammad K, et al. (2010) Consumption of fruit and vegetables among elderly people: a cross sectional study from Iran. Nutr J 9, 2

15. Hall JN, Moore S, Harper SB, et al. (2009) Global variability in fruit and vegetable consumption. Am J Prev Med 36, $402 \mathrm{e} 5-409 \mathrm{e} 5$.

16. Subar AF, Heimendinger J, Patterson BH, et al. (1995) Fruit and vegetable intake in the United States: the baseline survey of the Five A Day for Better Health Program. Am J Health Promot 9, 352-360.

17. Blanck HM, Gillespie C, Kimmons JE, et al. (2008) Trends in fruit and vegetable consumption among U.S. men and women, 1994-2005. Prev Chronic Dis 5, A35.

18. Blake M, Chaudhury M, Deverill C, et al. (2004) Health Survey for England 2003. Vol. 2: Risk Factors for Cardiovascular Disease. London: The Stationery Office.

19. Estaquio C, Druesne-Pecollo N, Latino-Martel P, et al. (2008) Socioeconomic differences in fruit and vegetable consumption 
among middle-aged French adults: adherence to the 5 A Day recommendation. J Am Diet Assoc 108, 2021-2030.

20. Tamers SL, Agurs-Collins T, Dodd KW, et al. (2009) US and France adult fruit and vegetable consumption patterns: an international comparison. Eur J Clin Nutr 63, 11-17.

21. Kanungsukkasem U, Ng N, Van Minh H, et al. (2009) Fruit and vegetable consumption in rural adults population in INDEPTH HDSS sites in Asia. Glob Health Action 2.

22. Jaime PC \& Monteiro CA (2005) Fruit and vegetable intake by Brazilian adults, 2003. Cad Saude Publica 21, Suppl., $19-24$.
23. Jaime PC, Figueiredo IC, Moura EC, et al. (2009) Factors associated with fruit and vegetable consumption in Brazil, 2006. Rev Saude Publica 43, Suppl. 2, 57-64.

24. Thompson B, Demark-Wahnefried W, Taylor G, et al. (1999) Baseline fruit and vegetable intake among adults in seven 5 a day study centers located in diverse geographic areas. J Am Diet Assoc 99, 1241-1248.

25. Roos G, Johansson L, Kasmel A, et al. (2001) Disparities in vegetable and fruit consumption: European cases from the north to the south. Public Health Nutr 4 , $35-43$. 